\title{
A Laboratory-Scale Instrumented Forging Hammer as an Intermediate Strain Rate Testing Device
}

\author{
Julen Agirre ${ }^{1 *}$, Borja Erice $^{1,2}$, David Abedul ${ }^{1}$, Eneko Saenz de Argandoña ${ }^{1}$, Nagore Otegi ${ }^{1}$, \\ Lander Galdos ${ }^{1}$ \\ ${ }^{1}$ Department of Mechanics and Industrial Production, Mondragon Unibertsitatea, \\ Loramendi 4, 20500 Mondragon, Spain \\ ${ }^{2}$ IKERBASQUE, Basque Foundation for Science, Bilbao, Spain
}

\begin{abstract}
Mechanical characterisation of metallic materials at intermediate strain rates is essential to calibrate and validate computational models for industrial applications such as high-speed forming processes i.e. hammer forging, blanking, forming, etc. The most common devices that perform medium to high loading rate experiments are the servo-hydraulic universal testing machines and Split Hopkinson bar systems. Here we analyse the possibility of employing an in-house designed and constructed DirectImpact Drop Hammer (DIDH) for material mechanical characterisation at medium strain rates, ranging from 100 to $300 \mathrm{~s}^{-1}$. To show the suitability of the DIDH for mechanical characterisation, uniaxial compression experiments on S235JR structural steel are conducted and compared with finite element (FE) simulations performed with an elasticthermoviscoplastic material model previously calibrated with Split Hopkinson Pressure Bar (SHPB) tests.
\end{abstract}

\section{Introduction and motivation}

Metal forming in its multiple variants (forging, forming, blanking, etc.) is massively employed in the automotive, the aeronautical or the oil and gas industries, when certain mechanical properties such as high strength and ductility are required, as they are very costeffective operations for large series production. These operations however are performed at loading rates that exceed by far the quasi-static regime. The strain rates that are achieved by the material are roughly on the order of 10 to $300 \mathrm{~s}^{-1}$, what will be referred henceforth as the intermediate strain rate regime. It is therefore essential to be able to mechanically characterise the metallic alloys heavily employed by these industrial sectors. However, testing within this range using universal testing machines equipped with conventional load cells becomes extremely challenging since they often have a limited stroke velocity. If such velocity is not an issue, then inertia effects and wave propagation become more relevant making the load measurements more troublesome. The most used equipment for dynamic testing is the wellknown SHPB system, but it also has certain limitations when it comes to intermediate strain rate testing: (i) the maximum displacement achievable by the system to deform or fracture the specimen and (ii) the validity of the force readings due to wave overlapping. For a given strain rate, both limitations are fully defined by the geometry and configuration of the system. Therefore, the lower the strain rate the larger the system has to be. In general, this is limited by laboratory space and resource availability. Different solutions to avoid or circumvent these

*Corresponding author: jagirreb@mondragon.edu 
issues have been proposed in the literature. Some of them propose to cleverly modify the system's configuration or redesign certain elements [1-2], whereas others prefer to rely on the manipulation of the strain gauge signals even if overlapping exist [3-5]. As an alternative to the SHPB systems for intermediate strain rate testing, we present an in-house designed and constructed Direct Impact Drop Hammer (DIDH). To show the suitability of this device for mechanical characterisation, compression experiments on S235JR structural steel are conducted and compared with finite element FE simulations. The steel is modelled with a Johnson-Cook-type material model calibrated using quasi-static and SHPB tests.

\section{Direct-Impact Drop Hammer}

Considering the difficulties to perform intermediate strain rate tests employing universal hydraulic commercial testing devices and SHPB systems, an automatized in-house Automatic ThermoMechanical Tester (ATMT) was designed and constructed. This testing machine is capable of reproducing a wide range of real hot and cold industrial metal forming conditions, from relatively low strain rates $\left(<1 \mathrm{~s}^{-1}\right)$ attained for example in processes with hydraulic presses up to elevated strain rates $\left(>100 \mathrm{~s}^{-1}\right)$ reached in hammer forging processes. This novel apparatus, shown in Figure 1, was designed to be able to test diverse forming strategies, including intermediate heating cycles combined with different loading rate scenarios. The ATMT is composed of four main sections: a heating furnace, an isothermal hydraulic press, a cooling system and the DIDH. To reduce human errors as much as possible, the apparatus is fully automatized and the sample manipulation is carried out by an electropneumatic arm that is integrated in the machine.

The mechanical module of the DIDH is comprised of a $92 \mathrm{~kg}$ hammer that is pushed by a high-pressure pneumatic cylinder propelling it towards the anvil, the fixed part of the system. The hammer can be set-up at different heights which allows for impact velocities to range from 2.5 up to $5 \mathrm{~m} / \mathrm{s}$ and a maximum applicable deformation energy of $1.15 \mathrm{~kJ}$. The anvil is instrumented with a piezoelectric force sensor (Kistler 9106A) allowing for direct force measurements.

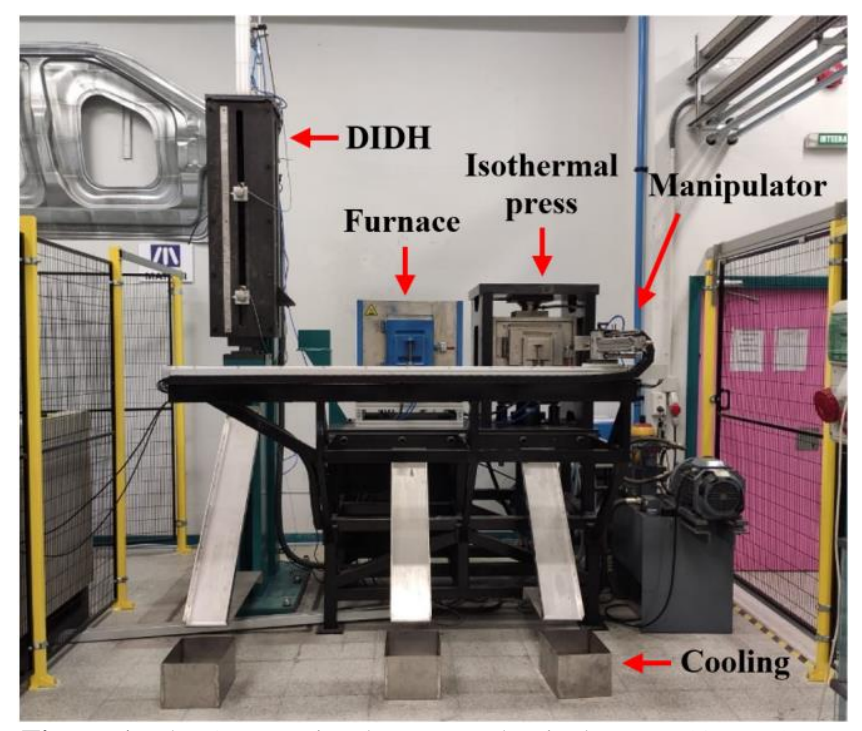

Figure 1. The Automatic ThermoMechanical Tester (ATMT) 


\section{Computational material model}

\subsection{Constitutive equations}

The incremental stress-strain relationship of the linear elastic isotropic law defined assuming the additive decomposition of the strain tensor, in terms of its elastic $\left(\boldsymbol{\varepsilon}^{e}\right)$ and plastic $\left(\boldsymbol{\varepsilon}^{p}\right)$ parts, as:

$$
\dot{\boldsymbol{\sigma}}=\mathbb{C}: \dot{\boldsymbol{\varepsilon}}^{e}=\mathbb{C}:\left(\dot{\boldsymbol{\varepsilon}}-\dot{\boldsymbol{\varepsilon}}^{p}\right)
$$

where $\mathbb{C}$ is the elastic stiffness tensor [6], that contains the elastic modulus $E=200 \mathrm{GPa}$ and the Poisson's ratio $v=0.3$. The von Mises yield function is chosen to describe the plastic yielding and is given by:

$$
f[\boldsymbol{\sigma}, p, \dot{p}, T]=\bar{\sigma}[\boldsymbol{\sigma}]-k[p, \dot{p}, T]
$$

where $\bar{\sigma}=\sqrt{\frac{3}{2} \mathbf{S}: \mathbf{S}}$ is the von Mises stress, $\mathbf{s}=\operatorname{dev}[\boldsymbol{\sigma}]$ is the deviatoric stress tensor, $k$ is the flow stress of the material, $p=\int \dot{p} d t$ is the equivalent plastic strain, $\dot{p}$ is the equivalent plastic strain rate and $T$ is the temperature. The work hardening of the material is described with a Johnson-Cook-type law, where the strain rate and temperature effects are considered to be independent from each other,

$$
k[p, \dot{p}, T]=\left\{\sigma_{0}+\sum_{i=1}^{2} Q_{i}(1-\exp [-C p])\right\}\left\{1+C \ln \left[\frac{\dot{p}}{\dot{p}_{0}}\right]\right\}\left\{1-\left(\frac{T-T_{r}}{T_{m}-T_{r}}\right)^{m}\right\}
$$

where $\sigma_{0}$ is the yield stress, $Q_{1}, C_{1}, Q_{2}, C_{2}$ are the constants of the two-term Voce strain hardening law, $C$ is the strain rate coefficient, $\dot{p}_{0}=10^{-3} \mathrm{~s}^{-1}$ is the reference strain rate, $m$ is the thermal softening exponent, $T_{r}=25{ }^{\circ} \mathrm{C}$ is the refence temperature and $T_{m}=1500{ }^{\circ} \mathrm{C}$ is the melting temperature. The flow rule is chosen to be associative,

$$
\dot{\boldsymbol{\varepsilon}}^{p}=\dot{p} \frac{3}{2} \frac{\mathbf{s}}{\bar{\sigma}}
$$

The evolution equation for the temperature during adiabatic heating conditions is assumed to be:

$$
\dot{T}=\frac{\chi}{\rho C_{p}} \boldsymbol{\sigma}: \dot{\boldsymbol{\varepsilon}}^{p}
$$

where $\rho=7850 \mathrm{~kg} / \mathrm{m} 3$ is the mass density, $C_{p}=460 \mathrm{~J} /\left(\mathrm{kg} \cdot{ }^{\circ} \mathrm{C}\right)$ is the specific heat $\chi=0.9$ and is the Taylor-Quiney coefficient. The constitutive equations are implemented in their incremental form as a user material subroutine in the LS-DYNA non-linear explicit time integration FE solver as in [6].

\subsection{Constant identification}

Quasi-static uniaxial tension (UT) and quasi-static and high-rate uniaxial compression (UC) tests were conducted on the S235JR low carbon content ferritic-pearlitic structural steel to calibrate the work hardening of the computational material model and to estimate the friction coefficient between the platens/bars and the specimen in the case of the UC tests. UT specimen was milled out from a $20 \mathrm{~mm}$ thick plate and its geometry followed the EN ISO 6892-1 standard having a gauge length of $L=50 \mathrm{~mm}$. The UC specimens were cylinders of diameters of 13.5 and $9.9 \mathrm{~mm}$ for the quasi-static and high-rate tests respectively. The length of the former was $20 \mathrm{~mm}$, while the length of the latter was $6.87 \mathrm{~mm}$. 
The quasi-static tests were performed in an Instron-4206 hydraulic universal testing machine equipped with a $100 \mathrm{kN}$ load cell at a strain rate of $10^{-3} \mathrm{~s}^{-1}$ averaged over the gauge length of the specimens. In the case of the UT tests the engineering strains were calculated from a $50 \mathrm{~mm}$ gauge extensometer located within the gauge area, while in the UC experiments was calculated tracking the compression platens.

A SHPB was employed to perform the high-rate UC tests. It follows the same setup and configuration as that described in [7]. The striker was launched at three velocities 5.8, 8.3 and $10.7 \mathrm{~m} / \mathrm{s}$ to obtain different strain rates. These velocities were measured with a laser right before the striker impacted the input bar. The specimen length history $\Delta L[t]$ was computed processing the $384 \times 288 \mathrm{px}^{2}$ resolution images taken with a Phantom v2511 high-speed camera at $150 \mathrm{kfps}$ with the point tracking technology available in the eCorr v4 software. The force histories were measured directly from the transmitted strain wave $\left(\varepsilon_{t}\right)$ red from the output bar strain gauge station as $F[t]=A_{b} E_{b} \varepsilon_{t}[t]$ where $E_{b}$ are $A_{b}$ the elastic modulus and cross-section area of the bar respectively.

The axial engineering strain $(e)$ and stress $(s)$ histories of all the experiments were calculated as,

$$
e[t]=\Delta L[t] / L \text { and } s[t]=F[t] / A
$$

The strain hardening constants were identified through inverse modelling of the quasi-static UT tests with an iterative domain reduction scheme available in the LS-OPT optimisation software package (see Table 1). The resulting engineering stress-strain curve is compared against the experiments in Figure 2 (a) showing an excellent agreement. Since the study was focused on the compressive mechanical behaviour of the material, the compression tests at several loading rates were simulated to calibrate the strain rate and temperature effects as well as to estimate the friction coefficient ( $\mu=0.3$ ) by measuring and comparing the final geometry of the specimens to the FE simulations. The engineering stress-strain curves obtained from the numerical simulations were in good agreement with the experiments as it can be seen in Figure 2 (b).

Table 1. Johnson-Cook material constants for the S235JR low-alloy steel.

\begin{tabular}{ccccccc}
\hline$\sigma_{0}[\mathrm{MPa}]$ & $Q_{1}[\mathrm{MPa}]$ & $C_{1}$ & $Q_{2}[\mathrm{MPa}]$ & $C_{2}$ & $C$ & $m$ \\
250.00 & 105.00 & 35.24 & 330.00 & 30.00 & 0.06 & 0.35 \\
\hline
\end{tabular}
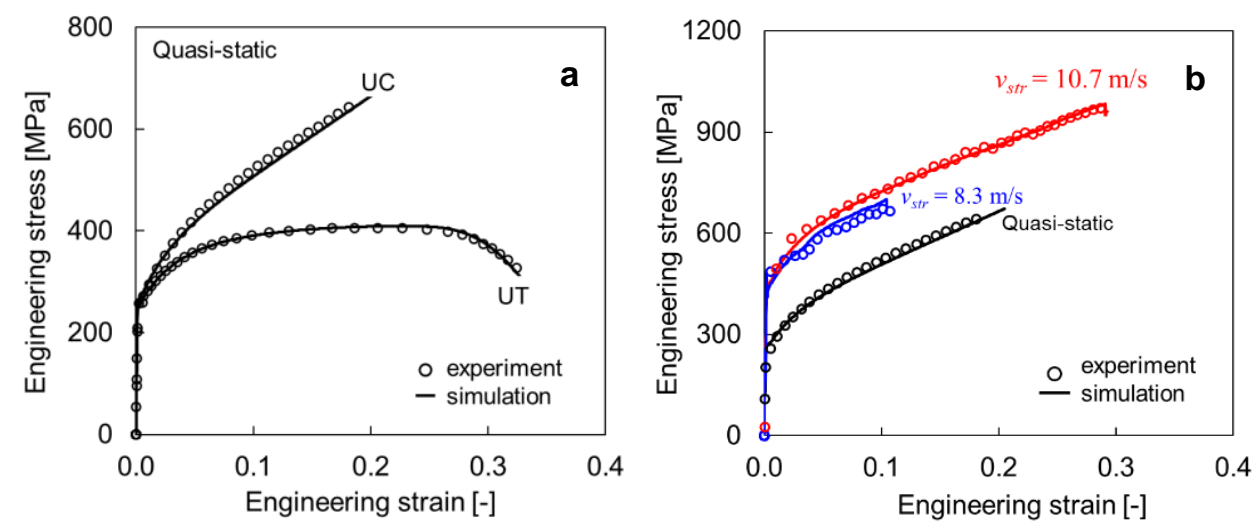

Figure 2. (a) Quasi-static engineering stress-strain compression and tension curves compared to the numerical predictions. (b) Uniaxial compression tests against the numerical mechanical response for several loading rates. 


\section{Validation}

The DIDH, introduced in section 2, was employed to perform UC tests of S235JR steel at different loading rates. Specimens with the same geometry as that used in the quasi-static UC tests were tested at hammer impact velocities of 3.3, 4.0, 4.7 and $5.0 \mathrm{~m} / \mathrm{s}$ to obtain different strain rates. Three repetitions were performed for each of the four hammer impact velocities where a very low deviation among each group was observed. The tests were recorded with a Photron Fastcam-APX RS250K high-speed camera (see Figure 1) that was set up at $15 \mathrm{kfps}$ with a resolution of $256 \times 512 \mathrm{px}^{2}$. A coarse random grid of the white dots on a dark background was generated on the hammer (moving) and on the anvil (static) surfaces for subsequent Digital Image Correlation (DIC) analysis. The specimen length history was obtained from the displacement histories of the dies postprocessing the high-speed camera images with GOM Correlate DIC commercial software package. The engineering strain and strain rate histories were calculated as in the set of equations (6). The force sensor mounted directly in the anvil was employed in combination with a Tektronix TSD 2004B oscilloscope with an acquisition frequency of $300 \mathrm{kHz}$ to obtain the force histories.

The DIDH is not able to maintain a relative constant strain rate unless the energy invested into deforming the specimen is way smaller than that generated by the drop hammer. In this particular case, as shown in Figure 3, the strain rate decays as the specimen gets compressed, which is directly related to the loss of velocity of the hammer. It is the objective of the current investigation to demonstrate that although these particular tests do not provide a constant strain rate, as is typically the case in the universal testing and SHPB devices, they can be used for calibration purposes as long as the displacement histories are properly monitored.
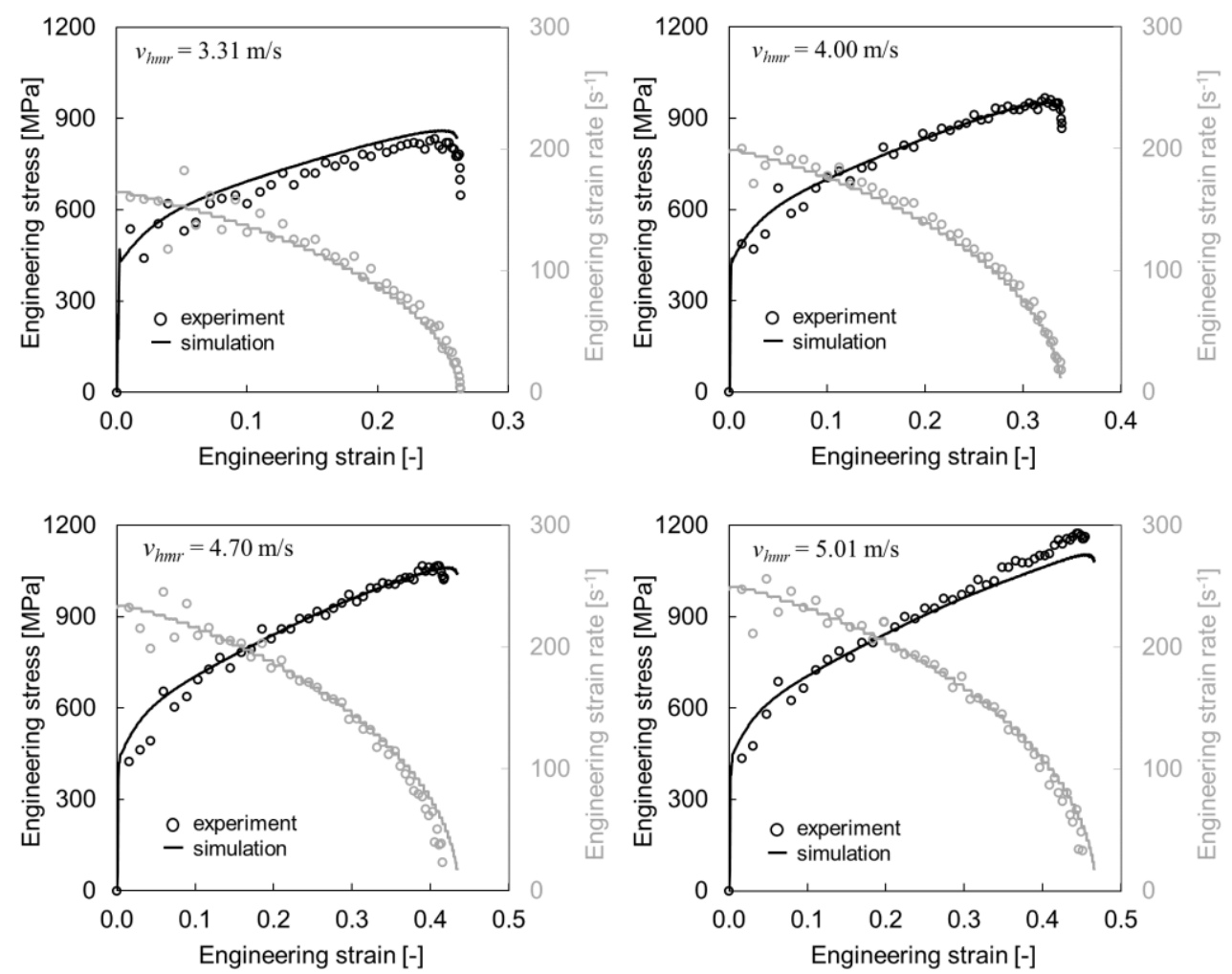

Figure 3. Engineering stress-strain and engineering strain rate-strain curves for the four impact velocities compared with their corresponding numerical responses. 
Numerical simulations of the DIDH tests with the calibrated material model for the S235JR steel were carried out with LS-DYNA FE solver. The specimens were discretised with eightnode reduced integration elements with hourglass control that had same size as that used in the calibration procedure. The same friction coefficient as that calculated in section 3 was applied between the dies, modelled as rigid walls, and the specimen. The DIC measured displacement histories were applied as prescribed motions in the rigid walls. The engineering stress-strain and strain rate-strain curves shown in Figure 3, following the same procedure as that used in the experiments. Apart from being in excellent agreement, the FE simulations show that if the displacement histories are properly measured, as is the case here, these stressstrain curves could be used for calibration purposes.

\section{Conclusions}

A new experimental device focused on the mechanical characterisation at intermediate strain rates for metal forming applications has been presented. To validate the suitability the apparatus, S235JR steel UC tests have been conducted in the DIDH and FE simulations of such tests have been performed with a Johnson-Cook-type material model that has been calibrated with quasi-static and SHPB tests. The results show that the DIDH can be used for mechanical characterisation at intermediate strain rates as long as the displacement history of the hammer is properly measured.

The authors would like to acknowledge the financial support of the Basque Government through the projects OGForge and DIGICUT. Special thanks to ULMA Forja S. Coop. and Fagor Arrasate S. Coop. for the financial and technical support. The authors would also like to thank the support of the Centre of Advanced Structural Analysis (CASA) (Project No. 237885 funded by the Research Council of Norway) at the Norwegian University of Science and Technology (NTNU).

\section{References}

1. G. Gary, D. Mohr, Modified Kolsky Formulas for an Increased Measurement Duration of SHPB Systems, Experimental Mechanics, 53, 713-717 (2013).

2. W.R. Whittington, A.L. Oppedal, D.K. Francis, M.F. Horstemeyer, International Journal of Impact Engineering, 81, 1-7 (2015).

3. J. Shim, D. Mohr, International Journal of Impact Engineering, 36, 1116-1127 (2009).

4. M. Bussac, P. Collet, G. Gary, O. Ramzi, An optimisation method for separating and rebuilding one-dimensional dispersive waves from multi-point measurements. Application to elastic or viscoelastic bars, Journal of the Mechanics and Physics of Solids, 50, 321-349 (2002).

5. B. Song, W. Chen, W.Y. Lu, Mechanical characterization at intermediate strain rates for rate effects on an epoxy syntactic foam, International Journal of Mechanical Sciences, 49, 1336-1343 (2007).

6. J.C. Simo, T. Hughes, Computational Ineslasticity, Springer-Verlag (1998).

7. J. F. Berntsen, D. Morin, A. H. Clausen, M. Langseth, Experimental investigation and numerical modelling of the mechanical response of a semi-structural polyurethane adhesive, International Journal of Adhesion and Adhesives, 95, 102395 (2019). 